\title{
Laser modulation simulation of micro- crack morphology evolution during chemical etching
}

Chen, Zhi, Xiao, Huapan, Li, Zhibin, Yu, Na, Wang, Hairong, et al.

Zhi Chen, Huapan Xiao, Zhibin Li, Na Yu, Hairong Wang, Rongguang Liang, "Laser modulation simulation of micro-crack morphology evolution during chemical etching," Proc. SPIE 11063, Pacific Rim Laser Damage 2019: Optical Materials for High-Power Lasers, 110631H (8 July 2019); doi: $10.1117 / 12.2540729$

SPIE Event: Pacific Rim Laser Damage 2019 and Thin Film Physics and Applications 2019, 2019, Qingdao, China 


\title{
Laser modulation simulation of micro-crack morphology evolution during chemical etching
}

\author{
Zhi Chen ${ }^{\mathrm{a}, \mathrm{b}}$, Huapan Xiao ${ }^{\mathrm{a}, \mathrm{c}}$, Zhibin $\mathrm{Li}^{\mathrm{a}, \mathrm{b}}, \mathrm{Na} \mathrm{Yu}^{\mathrm{a}, \mathrm{b}}$, Hairong Wang ${ }^{\text {a, b }}$, Rongguang Liang ${ }^{\mathrm{c}}$ \\ ${ }^{a}$ State Key Laboratory for Manufacturing Systems Engineering, Xi'an Jiaotong University, Xi'an, \\ Shaanxi 710049, China; ' $S$ School of Mechanical Engineering, Xi'an Jiaotong University, Xi'an, \\ Shaanxi 710049, China; ${ }^{\mathrm{c} C o l l e g e}$ of Optical Sciences, University of Arizona, Tucson, Arizona \\ 85721, USA \\ *whairong@mail.xjtu.edu.cn
}

\begin{abstract}
Subsurface micro-cracks will be generated during the grinding and polishing processes of optical components. Microcracks have a modulation effect on the laser, thereby reducing the laser damage threshold. The FDTD method is used to simulate the light intensity distribution modulated by micro-crack. By comparing the simulation results of radial crack, parabolic crack and elliptic crack, the modulation mechanism of micro-crack is revealed. The results show that for the crack with the same width and depth, light intensity enhancement factor (LIEF) modulated by radial crack on the rear surface and parabolic crack on the front surface is the largest; LIEF modulated by elliptical crack on the rear surface and radial crack on the front surface is the smallest. In addition, when the crack width-depth ratio is the same, the larger the depth, the higher the LIEF. As the width-depth ratio increases, the LIEF value increases firstly, then decreases, and finally approaches a stable value.
\end{abstract}

Keywords: FDTD, parabolic crack, radical crack, elliptical crack, light intensity enhancement factor

\section{INTRODUCTION}

With the development of high peak power laser, the surface quality requirements for optical components are getting higher and higher ${ }^{1}$. However, subsurface defects may exist in optical components during their processing such as grinding and polishing, which seriously affects the laser damage resistance of optical components ${ }^{2}$. The main reason is that the discontinuity of the crack wall ${ }^{3}$ or the interference between the incident beam and the reflected beam ${ }^{4}$ make the local light intensity near the micro-crack strengthen. For the research of the light field modulation mechanism of subsurface micro-cracks, many scholars at home and abroad have done a lot of work, but they mainly focus on the modulation simulation of three kinds of typical micro-cracks (radial crack, lateral crack and conical crack) ${ }^{2}$. Little research has been done on the mechanism of micro-cracks with other morphologies.

In recent years, chemical etching is used in the post-processing of optical components. Chemical etching can expose subsurface micro-cracks and evolve their morphologies ${ }^{5-6}$. Most studies show that slight chemical etching can improve the laser damage resistance of optical components ${ }^{7-8}$. However, the mechanism that the morphological changes of chemical etched micro-cracks improve the laser damage resistance of optical elements has not been reported.

In this work, subsurface micro-cracks are observed by laser scanning confocal microscope (OLS4000) and their profiles were extracted. Then the models of three kinds of micro-crack (radial crack, parabolic crack and elliptical crack) are applied to simulate the light intensity distribution by using two-dimensional finite difference time domain (2D-FDTD) method. The modulation mechanism of micro-crack is studied by comparing the light intensity distribution modulated by three kinds of cracks on the front and rear surfaces. And the effect of the crack width-depth ratio is also considered. 


\section{EXPERIMENT AND MODEL}

\subsection{Experiment}

The polished $\mathrm{K} 9$ glass is chemically etched with a mixture of $\mathrm{HF}$ and $\mathrm{HNO}_{3}$ (volume ratio 4:1). The samples are cleaned at etching time of $10 \mathrm{~s}, 30 \mathrm{~s}, 1 \mathrm{~min}, 2 \mathrm{~min}$ and $4 \mathrm{~min}$, and observed under laser scanning confocal microscope. Figure 1 show the morphology evolution of the same group of micro-cracks at different etching time. Figure 2 is the profile evolution of crack No. 3. It can be found that with the increase of etching time, the length and width of micro-cracks increase, the depth decreases, and the width-depth ratio increases. Micro-cracks gradually change from slender to ellipsoid. It is found that the crack is parabolic. Based on this, a parabolic crack model is proposed (Fig. 3(b)). The modulation mechanism of parabolic crack on laser is studied by 2D-FDTD method, and the influence of width-depth ratio of parabolic crack on modulation is analyzed. In order to make a comparison, a radial crack model and an elliptical crack model are proposed.

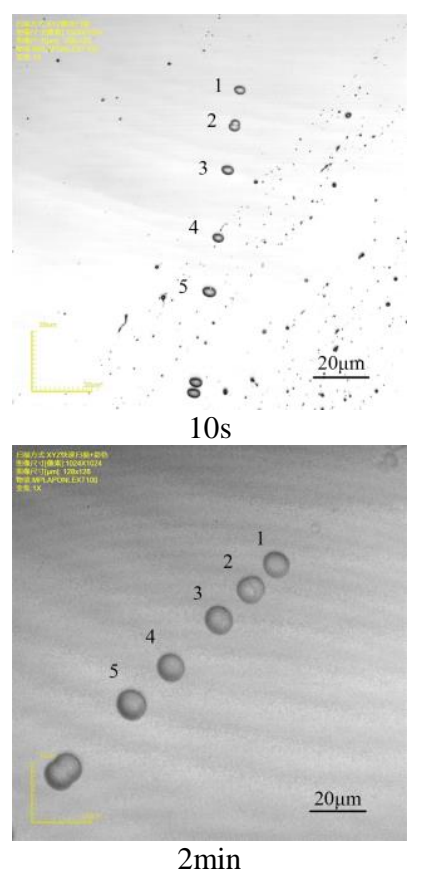

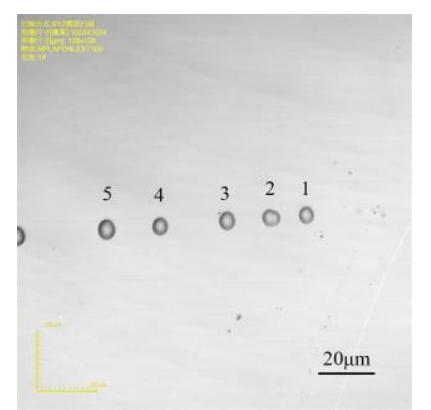

$30 \mathrm{~s}$

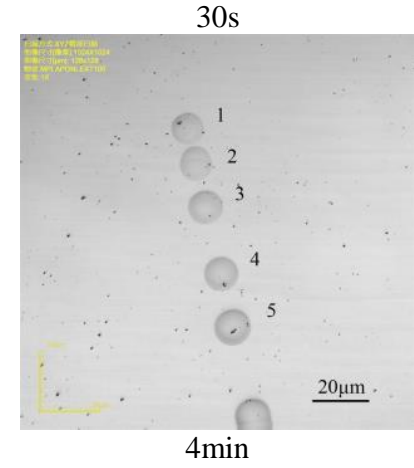

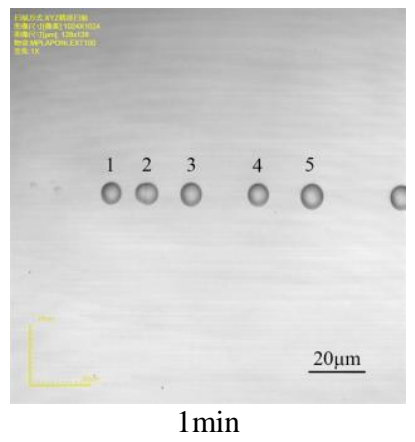

Figure 1. Morphology evolution of cracks in fused silica after chemical etching



Figure 2. Profile evolution of crack No. 3 in glass after chemical etching. 


\subsection{Model and method}

The simulation model for three kinds of crack, radial crack, parabolic crack and elliptical crack, is shown in Fig. 3. And formula (1) is the equation of parabolic crack and formula (2) is the equation of elliptic crack. Here, a 2D model of crack is built under the irradiation of front-surface and rear-surface laser. The wavelength of incidence laser is $\lambda=1064 \mathrm{~nm}$. For ensuring the calculation accuracy, the simulation region is meshed by the uniform rectangle with side length $\delta=\lambda / 28$ $=38 \mathrm{~nm}$, and the domain size is $600 \delta \times 480 \delta$. The perfectly matched layer (PML) absorbing-boundary condition ${ }^{7-8}$ is utilized in this work. In addition, the incident direction of laser beam is along the $y$ axis. The transverse electric (TE) wave is considered. The relative dielectric constants of glass and air are 2.25 and 1.0, respectively. The time step is determined by adjusting and observing the accurate light intensity distribution within the domain.

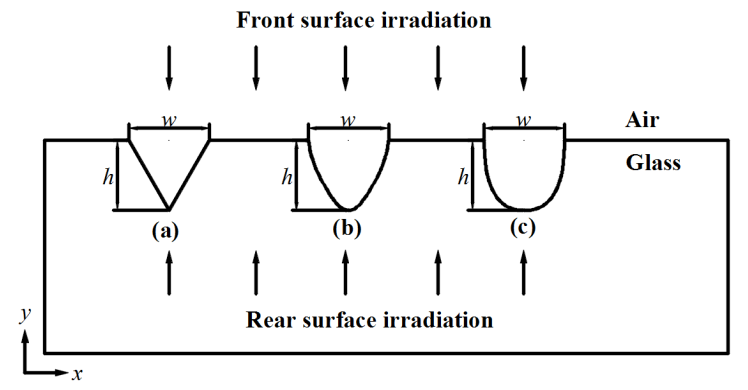

Figure 3. Schematic diagram of a simulation model: (a) radial crack, (b) parabolic crack, (c) elliptical crack.

$$
y=\frac{4 h}{w^{2}} x^{2}-h
$$

and

$$
\frac{4 x^{2}}{w^{2}}+\frac{y^{2}}{h^{2}}=1
$$

The refractive index of glass is $n=1.5$. The critical angle of total internal reflection is $41.8^{\circ}$. For the radial crack, parabolic crack and elliptical crack on the rear surface, the number of total internal reflections is related to the incident angle, defined as the angle between the incident wave and the normal of crack wall. And the relationship between the incident angle and the number of the total internal reflection is as Table $1^{9}$.

Table 1. The relationship between the number of total internal reflection and incident angle?

\begin{tabular}{cc}
\hline Incident angle $\theta$ & Number of total internal reflections \\
\hline $0<\theta<41.8^{\circ}$ & 0 \\
$41.8^{\circ} \leq \theta \leq 45^{\circ}$ & 1 \\
$45^{\circ}<\theta \leq 69.1^{\circ}$ & 2 \\
$\theta>69.1^{\circ}$ & 1 \\
\hline
\end{tabular}

In this paper, the modulation mechanism of three kinds of micro-cracks and the effect of width-depth ratio is mainly studied. The light intensity enhancement factor (LIEF) is introduced to quantitatively characterize the localized light intensity distribution caused by $\operatorname{crack}^{[2,4,10]}$. The LIEF is defined as maximal light intensity enhancement caused by cracks in the certain domain. 


\section{RESULTS AND DISCUSSION}

\subsection{Modulation by radial crack}

The simulation model for radial crack is shown in Fig. 3(a). Figures 4(a) and 4(b) show the light intensity distribution modulated by radial crack on the rear and front surfaces under TE mode illumination. The parameters of the radial crack include the crack depth $h=4 \lambda$ and the crack width $w=6 \lambda$. It can be seen that the distribution of LIEF modulated by radial crack on the front and rear surfaces is greatly different. When the radial crack is on the rear surface (Fig. 4(a)), the strong intensity domain around the crack is a series of hot spots parallel to the rear surface, and the maximum LIEF value is 9.86. Refer to the Table 1, because the angle between the crack and the surface is $\alpha=\arctan (2 h / w)=53.1^{\circ}$, the total internal reflection occurs when the incident beam reaches the crack wall. And when the reflected beam reaches to the rear surface, the incident angle is $73.8^{\circ}$, and the total internal reflection occurs again. Two total internal reflections occur during the whole process. The interference between reflected beam and incident beam results in an increase in light intensity.

When the radial crack is on the front surface (Fig. 4(b)), there are three strong intensity domains. The two are located inside the glass on the both sides of the crack symmetrically, and another is the discrete hot spot in the crack gap. The two strong intensity domains inside the glass are caused by the diffraction of the incident beam at the intersection points of the crack and the surface, and the interference with the light entering from the surface and the crack wall. The discrete hot spots in the crack gap is formed by the interference between the reflected beam from the crack wall and the incident beam. And the maximum LIEF value appears in the crack gap with a value of 3.49 .

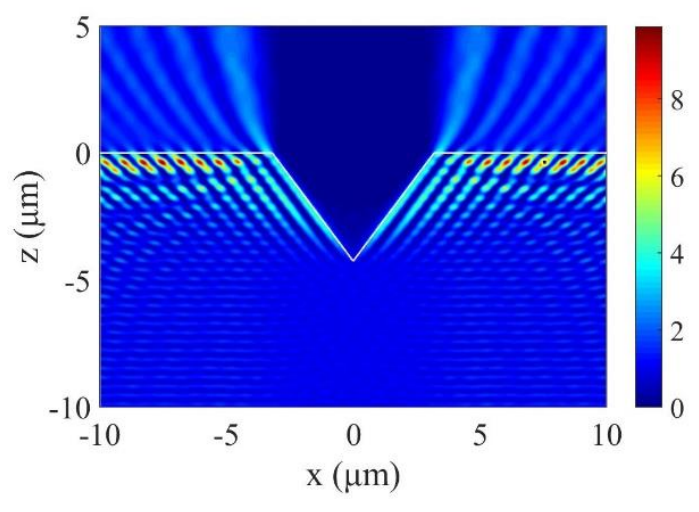

(a) on the rear surface



(b) on the front surface

Figure 4. Light intensity distribution modulated by the radial crack under the TE mode illumination.

Table 2. LIEF values modulated by three kinds of cracks.

\begin{tabular}{cccc}
\hline The incident surface & Radial crack & Parabolic crack & Elliptic crack \\
\hline Rear surface & 9.86 & 6.14 & 5.15 \\
Front surface & 3.49 & 5.43 & 4.64 \\
\hline
\end{tabular}

Considering the influence of radial crack width and depth on the light intensity modulation, five different kinds of crack depth ( $h$ is $1 \lambda, 2 \lambda, 3 \lambda, 4 \lambda$ and $5 \lambda$, respectively) are selected and the crack width $w$ is changed. The relationship between LIEF value and the width-depth ratio $\zeta(\zeta=w / h)$ of cracks is studied (Fig. 5).

From Fig. 5(a), it can be found that the relationship between LIEF and width-depth ratio of radial crack on the rear crack follow two laws: (1) the larger the depth at the same width-depth ratio, the higher the LIEF value. The main reason is that the larger the depth is, the longer the crack wall length is, the more light is received and the more reflected light is, which results in more interference between reflected light and incident light, and produces larger LIEF value; (2) With the increase of width-depth ratio, LIEF value increases firstly, then decreases, then increases gradually, and finally slowly decreases to a stable value. The difference of width-depth ratio leads to the change of the angle between the crack wall and the surface $(a=\arctan (2 h / w)=\arctan (2 / \zeta))$, and the angle $a$ is equal to the incident angle on the crack wall. Because the width-depth ratio is negatively correlated with the incident angle, the width-depth ratio $\zeta$ decreases gradually with the increase of the incident angle. When width-depth ratio $\zeta$ is about 2.237 , the incident angle is $41.8^{\circ}$, and the LIEF 
value is relatively small. With the increase of incident angle, the total internal reflection begins to appear, more light interferes with each other in the glass, and LIEF value increases. When width-depth ratio $\zeta$ is about 1.4 , the incident angle is $55^{\circ}$, and the LIEF reaches to the maximum value. Then with the increase of incident angle, LIEF decreases rapidly. When the width-depth ratio of radial crack is very small, the modulation effect of radial crack on light is slight, and the LIEF value is small, which has little effect on the damage threshold of optical glass.

Fig. 5(b) shows the relationship between LIEF and width-depth ratio of radial crack on the front crack. It can be found that with the increase of width-depth ratio, LIEF value increases firstly, then slowly decreases to a stable value. The LIEF value is relatively small, and the maximum value is not more than 4 . In addition, for radial crack at the same widthdepth ratio, the larger the depth, the higher the LIEF value.

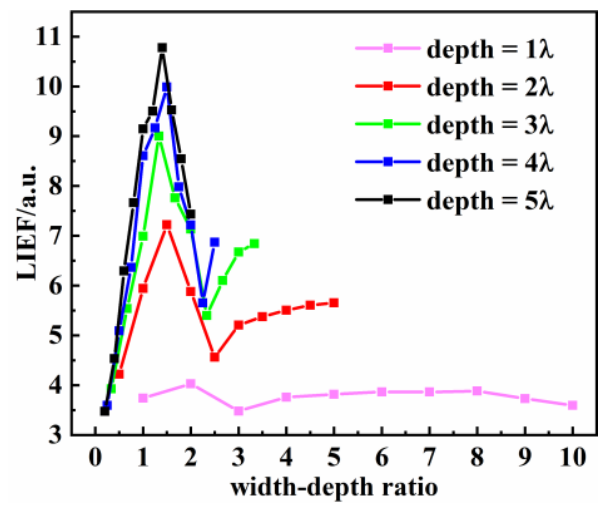

(a) radial crack on the rear surface

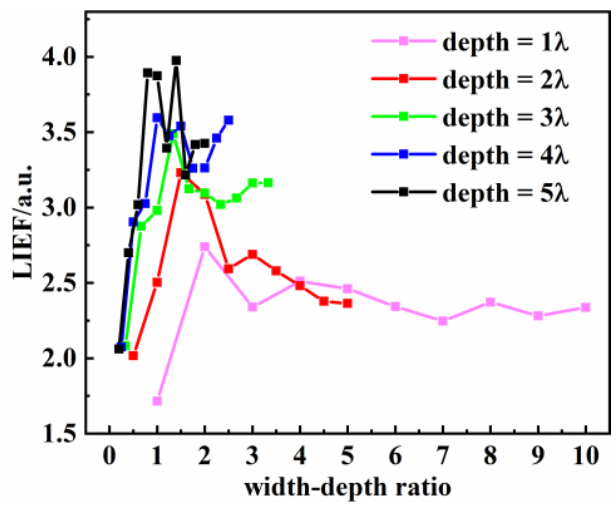

(b) radial crack on the front surface.

Figure 5. The relationship between LIEF value and the width-depth ratio $\zeta$ of radial crack.

\subsection{Modulation by parabolic crack}

The simulation model for parabolic crack is shown in Fig. 3(b). Figures 6(a) and 6(b) show the light intensity distribution modulated by parabolic crack on the rear and front surfaces under TE mode illumination. The parabolic crack has the same parameters as the radial crack. Compared with Fig. 4 and Fig. 6, it can be found that the light intensity distribution modulated by the parabolic crack on the front and back surfaces is similar to that of the radial crack. When the parabolic crack is on the rear surface (Fig.6 (a)), the hot spots also appear in the glass near the rear surface, and the LIEF value is 6.14, which is less than 9.86 of the radial crack (shown in Table 2). Because the crack wall of parabolic crack is a curved surface, the slope of the crack wall is not a fixed value. The incident angle of different regions on crack wall is different, and the light path is different from that under the radial crack, so the situation is more complicated. Figure 7 is a schematic diagram of the light path incident on a parabolic crack on the rear surface (symmetric about the central axis). When the incident light is in the a-d regions, the incident angle is less than $41.8^{\circ}$, no total reflection occurs, and some light passes through the crack wall and enters the air; when the incident light is in the d-e regions, the incident angle is between $41.8^{\circ}$ and $45^{\circ}$, one total internal reflection occurs; when the incident light is in the e-f regions, the incident angle is between $45^{\circ}$ and $69.1^{\circ}$, and two total internal reflections occur; when the incident light is in the remaining region, one total internal reflection occurs. Since some of the light in the a-d regions passes through the crack, less light is reflected by the crack wall than the radial crack, which participates in the interference enhancement. This is one reason why the LIEF modulated by the parabolic crack on the rear surface is smaller than that of the radial crack. At the same time, since the crack wall is a curved surface, the parabolic crack on the rear surface is equivalent to a convex mirror (except for partial transmission in some areas), which has a diverging effect on the incident light. And it causes the reflected light to be more divergent. The reflected light interferes with the incident light, so the hot spots are more dispersed, and the maximum LIEF value is relatively small. This is another reason why the LIEF modulated by the parabolic crack on the rear surface is smaller than that of the radial crack.

When the parabolic crack is on the front surface (Fig. 6(b)), there are also three strong intensity domains: two strong intensity bands on both sides of the crack in the glass and discrete hot spots in the crack gap. The maximum hot spot also appears in the crack gap, and the maximum LIEF value is 5.43, which is greater than that of the radial crack (shown in Table 2). The main reason is that the curve crack wall of the parabolic crack on the front surface is equivalent to a 
concave mirror with respect to the straight radial crack wall, which has a focus effect. So the light is concentrated in one place, and strong intensity is generated. And the focus point is closer in the bottom of the crack gap.

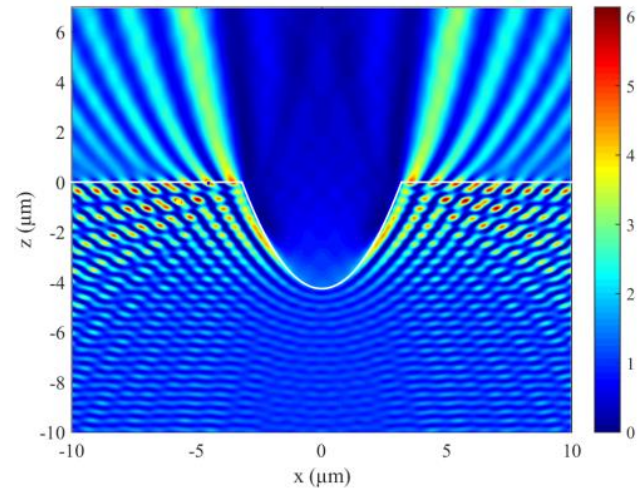

(a) on the rear surface

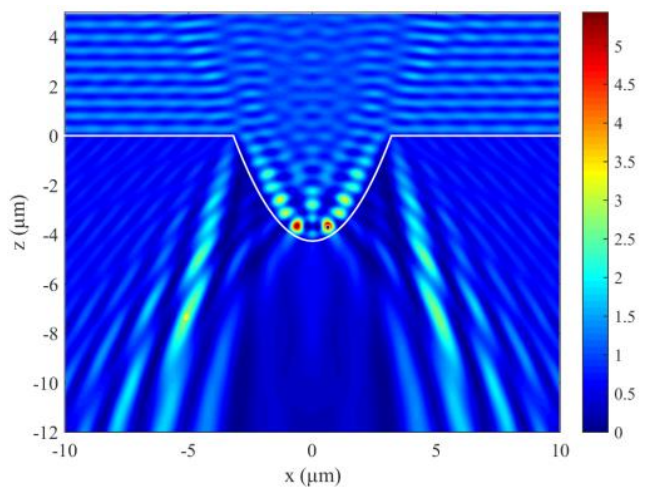

(b) on the front surface

Figure 6. Light intensity distribution modulated by the parabolic crack under the TE mode illumination.

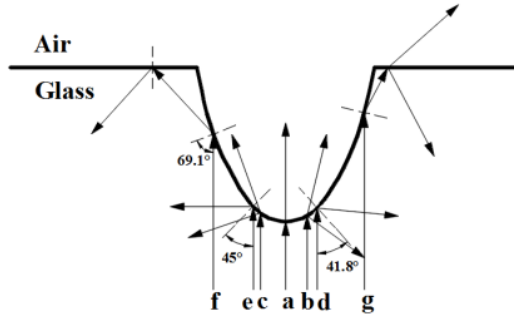

Figure 7. Schematic diagram of the light path incident on a parabolic crack on the rear surface.

Figure 8 shows the relationship between LIEF value and the width-depth ratio $\zeta(\zeta=w / h)$ of parabolic cracks. Like the radial cracks, five different kinds of crack depth ( $h$ is $1 \lambda, 2 \lambda, 3 \lambda, 4 \lambda$ and $5 \lambda$, respectively) are selected and the crack width $w$ is changed. It can be seen that the same trend can be found in all five kinds of crack depth, and the trend is the same as that of the relationship between LIEF and width-depth ratio of radial crack. For the parabolic crack on the rear surface (Fig. 8(a)), the difference is that the maximum LIEF value appears between the width-depth ratio of 2-2.5, and the maximum LIEF value is less than that of the radial crack with the same depth and width. For the parabolic crack on the front surface (Fig. 8(b)), the difference is that LIEF modulated by parabolic crack on the front surface is larger than that of radial crack.

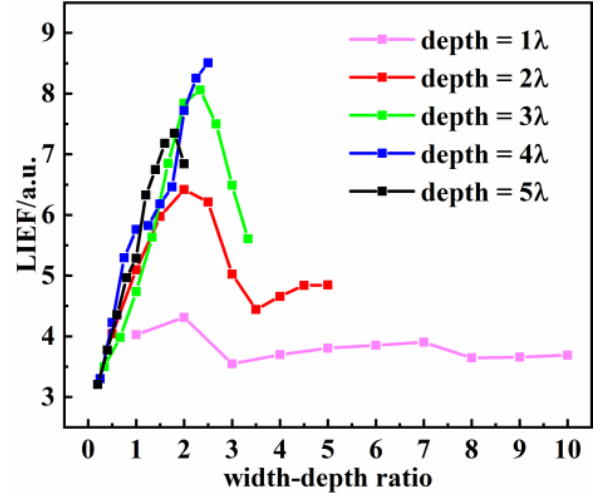

(a) parabolic crack on the rear surface

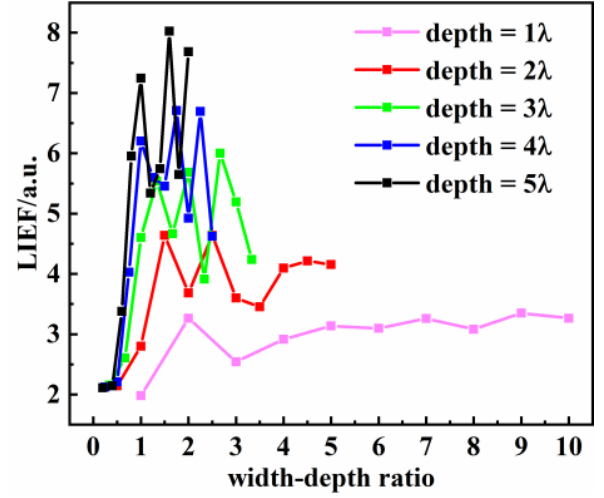

(b) parabolic crack on the front surface

Figure 8 . The relationship between LIEF value and the width-depth ratio $\zeta$ of parabolic crack. 


\subsection{Modulation by elliptical crack}

The simulation model for elliptical crack is shown in Fig. 3(c). Figures 9(a) and 9(b) show the light intensity distribution modulated by elliptical crack on the rear and front surfaces under TE mode illumination. The elliptical crack has the same parameters as the parabolic crack. The white dashed line in Fig. 8 is the contour of the parabolic crack with the same depth and width. Compared Fig. 6 with Fig. 9, it can be found that the light intensity distribution modulated by the elliptical crack on the front and back surfaces is very similar to that of the parabolic crack. When the elliptical crack is on the rear surface (Fig. 9(a)), the hot spots also appear in the glass near the rear surface, and the LIEF value is 5.15, which is less than that of the radial crack and the parabolic crack (shown in Table 2). Its modulation mechanism is similar to that of parabolic crack. Because the crack wall is a curved surface, which is equivalent to a convex mirror, it can diverge the incident light and the reflected light is more divergent, which leads to the scattering of the field intensity hot spots, and the maximum LIEF value is relatively small. From Fig. 9(a), it can be seen that the elliptic crack is more circular in contour than the parabolic crack, and the slope of the central part changes slowly, so the transmission area is larger and the reflected beam is less. In addition, the curvature of the elliptical crack changes more uniformly and the divergence effect is more obvious. Because of these two points, the LIEF modulated by the elliptic crack on the rear surface is less than that of parabolic crack.

When the elliptical crack is on the front surface (Fig. 9(b)), the light intensity distribution modulated by elliptical crack is basically the same as that of parabolic crack. The maximum hotspot also appears in the crack gap. The maximum LIEF value is 4.64, which is less than that of parabolic crack 5.43, but larger than that of radial crack 3.49 (shown in Table 2). Compared with the parabolic crack, the middle part of the elliptic crack is more flat, so the focus distance from the bottom of the crack is larger, but the maximum of LIEF is smaller than those of the parabolic crack.

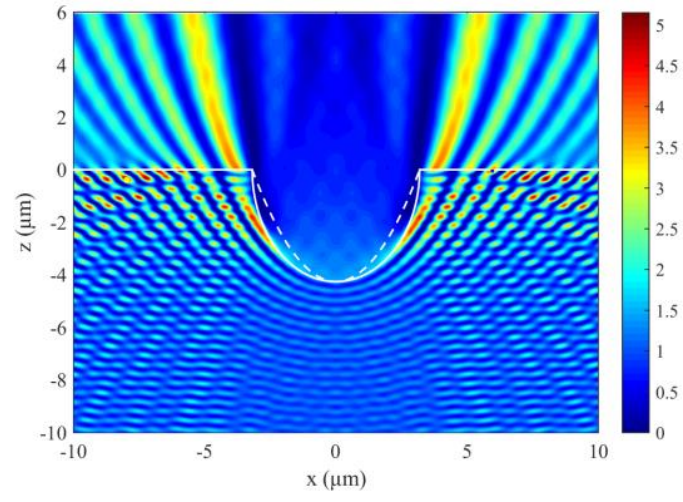

(a) on the rear surface

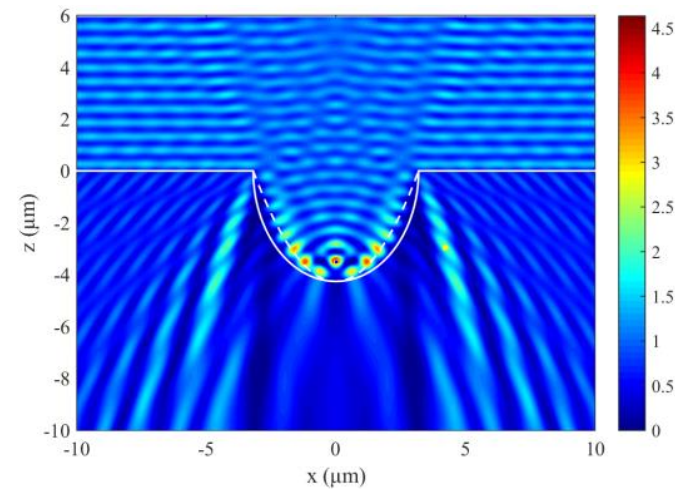

(b) on the front surface

Figure 9. Light intensity distribution modulated by the elliptical crack under the TE mode illumination.

Figure 10 is shown as the relationship between LIEF value and the width-depth ratio $\zeta(\zeta=w / h)$ of elliptical cracks. Like the radial cracks, five different kinds of crack depth ( $h$ is $1 \lambda, 2 \lambda, 3 \lambda, 4 \lambda$ and $5 \lambda$, respectively) are selected and the crack width $w$ is changed. It can be seen that the variation trend of LIEF with the width-depth ratio of elliptic crack is similar to that of parabolic crack. Among these three kinds of cracks with the same depth and width, the LIEF modulated by the elliptical crack on the rear surface is the smallest (Fig. 10(a)); the LIEF modulated by the elliptical crack on the front surface is between the radial crack and the parabolic crack (Fig. 10(b)). 


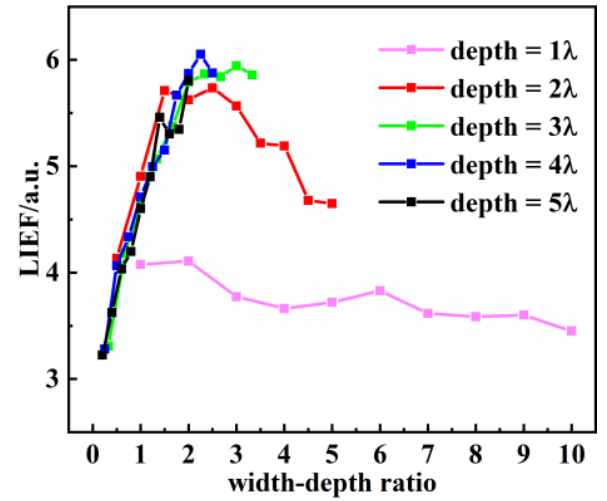

(a) elliptical crack on the rear surface

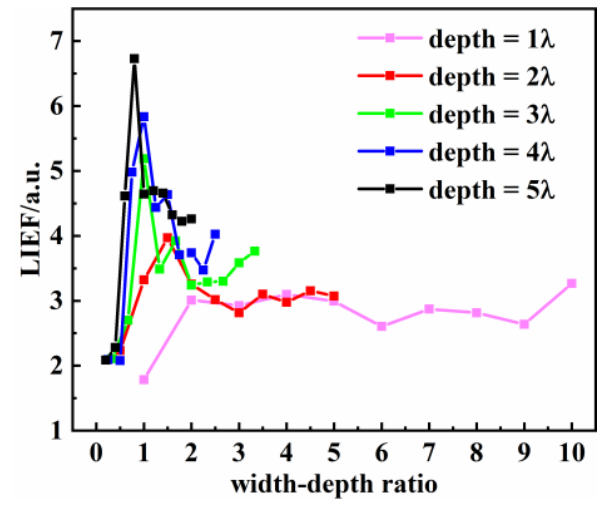

(b) elliptical crack on the front surface

Figure 10. The relationship between LIEF value and the width-depth ratio $\zeta$ of elliptical crack.

\section{CONCLUSIONS}

In this paper, the etching experiments show that with the increase of etching time, the single crack changes from "slender" to "ellipsoid", the length and width of cracks increase gradually, the depth decreases and the width-depth ratio decreases. The simulations about the light intensity distribution modulated by radial crack, parabolic crack and elliptical crack are conducted. The results show that for the crack with the same width and depth, light intensity enhancement factor (LIEF) modulated by radial crack on the rear surface and parabolic crack on the front surface is the largest; LIEF modulated by elliptical crack on the rear surface and radial crack on the front surface is the smallest. In addition, when the crack width-depth ratio is the same, the larger the depth, the higher the LIEF value. As the width-depth ratio increases, the LIEF value increases firstly, then decreases, and finally approaches a stable value. This work is to provide a reference for analyzing the effect of subsurface defects on laser-induced damage of optical components.

\section{ACKNOWLEDGMENT}

This work is supported by the National Natural Science Foundation of China (Grants No. 51175416 and No. 51675420), 111 Program (Grant No. B12016) and the National Key Research \& Development (R\&D) Program of China (Grant No. 2016YFB0501604-02). We appreciate the support from the International Joint Laboratory for Micro/Nano Manufacturing and Measurement Technologies.

\section{REFERENCES}

[1] Campbell, J. H., Hawley-Fedder, R. A., Stolz, C. J., Menapace J. A., Borden M. R., Whitman P. K., Yu J., Runkel M. J., Riley M. O., Feit M. D. and Hackel R. P., "NIF optical materials and fabrication technologies: an overview," Proc. SPIE 5341, 84-101 (2004).

[2] Zhang, L., Chen, W., Hu, L., "Systematic investigation on light intensification by typical subsurface cracks on optical glass surfaces," Appl. Opt. 52(5), 980-989 (2013).

[3] Bloembergen, N., "Role of cracks, pores, and absorbing inclusions on laser induced damage threshold at surfaces of transparent dielectrics," Appl. Opt. 12, 661-664 (1973).

[4] Génin, F. Y., Salleo, A., Pistor, T. V. and Chase, L. L., "Role of light intensification by cracks in optical breakdown on surfaces," Opt. Soc. Am. A 18, 2607-2616 (2001).

[5] Kolli, M., Hamidouche, M., Bouaouadja, N. and Fantozzi, G., "HF etching effect on sandblasted soda-lime glass properties," J. Eur. Ceram. Soc. 29, 2697-2704 (2009). 
[6] Ezz-Eldin, F. M., Abd-Elaziz, T. D. and Elalaily, N. A., "Effect of dilute HF solutions on chemical, optical, and mechanical properties of soda-lime-silica glass," J. Mater. Sci. 45, 5937-5949 (2010).

[7] Zheng, Z., Zu, X. T., Jiang, X. D., X. Xiang, Huang, J., Zhou, X. D., Li, C. H., Zheng, W. G. and Li, L., "Effect of HF etching on the surface quality and laser-induced damage of fused silica," Opt. Laser. Technol. 44, 10391042 (2012).

[8] Ye, H., Li, Y. G., Yuan, Z. G., Wang, J., Xu, Q. and Yang, W., "Improving UV laser damage threshold of fused silica optics by wet chemical etching technique," Proc. SPIE 9532, 1-6 (2015).

[9] Xiao, H. P., Wang, H. R., Chen, Z., Fu, G. L., and Wang, J. H., "Effect of brittle scratches on transmission of optical glass and its induced light intensification during the chemical etching," Opt. Eng. 56(10), 105101 (2017).

[10] Cheng, J., Chen, M., Liao, W., Wang, H., Xiao, Y., and Li, M., "Fabrication of spherical mitigation pit on $\mathrm{KH} 2 \mathrm{PO} 4$ crystal by micro-milling and modeling of its induced light intensification," Opt. Express 21(14), 16799-16813 (2013). 